\title{
Activation of Signaling Pathways Following Intestinal Resection: Turning It Up a Notch?
}

\author{
Yongjia Feng
}

Published online: 2 July 2013

(C) Springer Science+Business Media New York 2013

The intestinal tract is the most rapidly renewed mammalian tissue and thus has highly active stem and transit-amplifying cells that promote renewal of terminally differentiated cells that perform digestive functions. Notch signaling is thought to act directly on stem cells to promote their proliferation and also in progeny precursors to determine absorptive versus secretory terminal differentiation pathways, although Notch signaling may actually enhance cell differentiation with other signaling pathways such as the Wnt- $\beta$-catenin pathway promoting cell proliferation.

Short bowel syndrome (SBS) encompasses a spectrum of disorders that occur when malnutrition, weight loss, electrolyte abnormalities, severe diarrhea and failure to thrive result from insufficient absorptive areas of the small bowel. Following the experimental loss of functional small bowel surface area, compensatory adaptive responses, including increased intestinal epithelial cell (IEC) proliferation, crypt depth, and villous height occur through activation of the Hedgehog pathway [1] and Wnt- $\beta$-catenin signaling [2]. Little is known, however, regarding Notch signaling in this SBS adaptive response. In this issue, Chen et al. [3] implicate Notch signaling in intestinal adaptation following small bowel resection, a finding enhanced by the observation that the up-regulation of some of these factors occurs at a far earlier time than previously noted. Although Notch1 and Hes 1 were upregulated as early as $1 \mathrm{~h}$ after intestinal resection, the authors just observed expression changing early after resection, and they did not make a proof that these Notch changes were positively affecting SBS adaptation. The contribution of these changes towards

Y. Feng $(\bowtie)$

University of Michigan, Ann Arbor, MI, USA

e-mail: yongfeng@med.umich.edu
SBS adaption is unclear, since the adaptive response is thought to occur $48-72 \mathrm{~h}$ after resection.

Notch signaling is implicated in a plethora of developmental processes in an evolutionarily conserved fashion. Notch proteins are cell surface transmembrane receptors that mediate critically important cellular functions through direct cell-cell contact. Notch signaling, involved in many aspects of control of tissue homeostasis in a variety of adult tissues, regulates stem cell maintenance, cell differentiation and cellular homeostasis. There are four Notch genes, Notch1, 2, 3, and 4, and five Notch ligands, Jagged1, Jagged2, Delta1, Delta2, and Delta3, identified in vertebrates. Upon activation of Notch by its ligands, the Notch protein undergoes two proteolytic cleavages that release an intracellular Notch domain (NICD) facilitating its consequent translocation to the nucleus. In the nucleus, NICD interacts with the DNA binding protein and the co-activator Mastermind, promoting the transcription of the Notch target genes. In the current study, Hes1, a well-established Notch target gene, was unexpectedly upregulated very early ( $1 \mathrm{~h}$ after resection), without up-regulation of the Notch ligand Jagged-1, consistent with the involvement of other Notch ligands and target genes. As reported recently, the HES/HEY genes are not the only targets of Notch signaling; as in many tissues, loss of HES/HEY genes does not recapitulate all of the phenotypes of loss of Notch activity, indicating the existence of additional target genes. Indeed, there are a large number of cell-specific Notch targets, including myc and cyclinD [4]. These target genes of Notch can promote epithelial cell proliferation, and may be balanced cell cycle inhibitors such as p21, another Notch target. Since ligand activation of the Notch receptor is not entirely understood, future work will need to identify other ligands and targets involved in post-resection adaptation. 
Current data

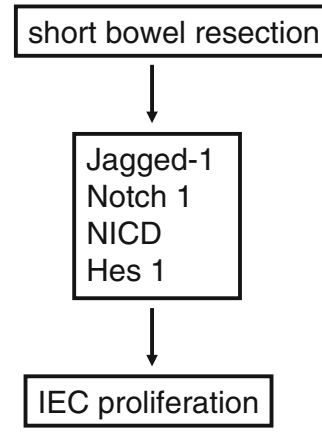

Future direction

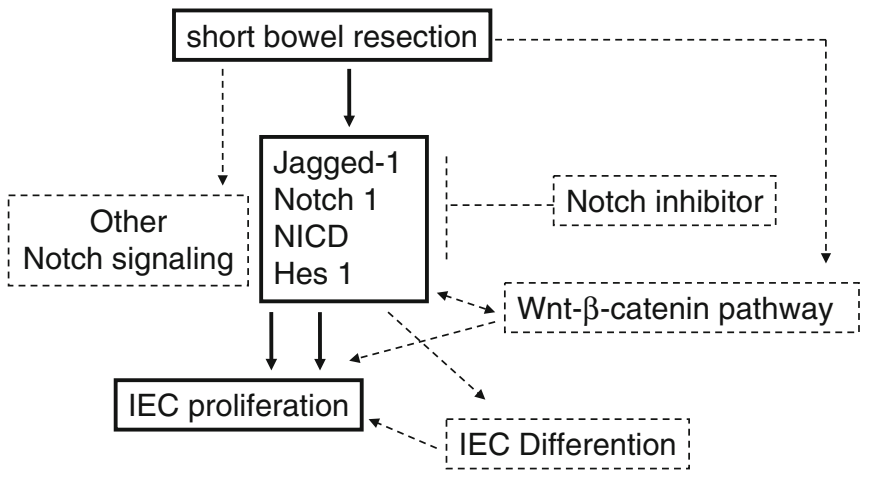

Fig. 1 Potential signaling pathways

The canonical Wnt signaling pathway is one of the major systems involved in the renewal of the intestinal epithelium $[5,6]$. Experimental manipulation of Wnt signaling influences IEC proliferation. Wnt signaling is integrated with Notch signaling in the intestinal epithelium, controlling stem cell turnover and epithelial regeneration. Using a novel $\beta$-catenin-inducible mouse model, Hirata et al. reported that $\beta$-catenin expression activated Notch signaling through upregulation of its ligands and receptors [7]; thus, a higher level of $\beta$-catenin activation is crucial for Notch activation. Notch1 and Notch2 receptors are expressed specifically in intestinal stem cells (ISC) [8], and the increased expression of Notch receptors could induce ISC-like cells by $\beta$-catenin induction. All this evidence showed a tight link between Wnt- $\beta$-catenin and Notch signaling. Although cell proliferation and Notch signaling were stimulated after small bowel resection in the accompanying study, the authors failed to demonstrate an obligatory cause-effect relationship between these two processes. In considering the importance of Notch in IEC differentiation, the observed elevated cell proliferation after bowel resection may result from activated Wnt- $\beta$-catenin signaling, while Notch signaling is activated to enhance cell differentiation of the rapidly expanding number of cell progenitors. To clarify this point, future work should include an examination of Wnt- $\beta$ catenin signaling expression at these early time points, as well as better co-localization of Jagged-1, NICD1, Hes-1 or $\beta$-catenin and PCNA positive cells in the stem cell region of the crypts. As mentioned, the authors only reported the upregulation of Jagged-1/Notch-1/Hes-1 and crypt cell proliferation after bowel resection. Additional studies, in particular Notch pathway blockade, could provide strong support for this hypothesis, in particular since the $\gamma$-secretase inhibitor dibenzazepine effectively inhibits Notch signaling in animal models $[9,10]$, along with neutralizing antibodies [11] or robust transgenic models.
In summary, the authors hypothesize that Notch signaling helps drive intestinal adaptation following small bowel resection. Figure 1 outlines potential signaling pathways that should be further explored in this very important clinical disease process.

\section{References}

1. Tang Y, Swietlicki EA, Jiang S, et al. Increased apoptosis and accelerated epithelial migration following inhibition of hedgehog signaling in adaptive small bowel postresection. Am J Physiol Gastrointest Liver Physiol. 2006;290:G1280-G1288.

2. Bernal NP, Stehr W, Zhang Y, Profitt S, Erwin CR, Warner BW. Evidence for active Wnt signaling during postresection intestinal adaptation. J Pediatr Surg. 2005;40:1025-1029; discussion 1029.

3. Chen G, Sun L, Yu M, et al. The Jagged-1/Notch-1/Hes-1 pathway is involved in intestinal adaptation in a massive small bowel resection rat model. Dig Dis Sci. (Epub ahead of print). doi: 10.1007/s10620-013-2680-3.

4. Krejci A, Bernard F, Housden BE, Collins S, Bray SJ. Direct response to Notch activation: signaling crosstalk and incoherent logic. Sci Signal. 2009;2:ra1.

5. Fevr T, Robine S, Louvard D, Huelsken J. Wnt/beta-catenin is essential for intestinal homeostasis and maintenance of intestinal stem cells. Mol Cell Biol. 2007;27:7551-7559.

6. Korinek V, Barker N, Willert K, et al. Two members of the Tcf family implicated in Wnt/beta-catenin signaling during embryogenesis in the mouse. Mol Cell Biol. 1998;18:1248-1256.

7. Hirata A, Utikal J, Yamashita S, et al. Dose-dependent roles for canonical Wnt signalling in de novo crypt formation and cell cycle properties of the colonic epithelium. Development. 2013;140:66-75.

8. Sato T, van Es JH, Snippert HJ, et al. Paneth cells constitute the niche for Lgr5 stem cells in intestinal crypts. Nature. 2011;469:415-418.

9. VanDussen KL, Carulli AJ, Keeley TM, et al. Notch signaling modulates proliferation and differentiation of intestinal crypt base columnar stem cells. Development. 2012;139:488-497.

10. van Es JH, van Gijn ME, Riccio O, et al. Notch/gamma-secretase inhibition turns proliferative cells in intestinal crypts and adenomas into goblet cells. Nature. 2005;435:959-963.

11. Wu Y, Cain-Hom C, Choy L, et al. Therapeutic antibody targeting of individual Notch receptors. Nature. 2010;464:1052-1057. 